\title{
Analysis of the Dynamic Response in the Railway Vehicles to the Track Vertical Irregularities. Part I: The Theoretical Model and the Vehicle Response Functions
}

\author{
M. Dumitriu \\ Department of Railway Vehicles, Faculty of Transport, University Politehnica of Bucharest, 313 Splaiul Independenţei, 060042, \\ Bucharest, Romania
}

Received 18 June 2015; Accepted 18 November 2015

\begin{abstract}
The paper herein focuses on the dynamic response of a two-bogie vehicle to the excitations derived from the track vertical irregularities. The symmetrical and antisymmetrical modes due from the bounce and pitch motions of the axles' planes in the two bogies are being considered. The analysis of the dynamic response in the vehicle relies on the response functions in three reference points of the carbody, composed by means of these response functions to the symmetrical and antisymmetrical excitation modes. Similarly, the dynamic response of the vehicle to the track stochastic irregularities is examined and expressed as a power spectral density of the carbody vertical acceleration and the root mean square of the acceleration and the index of the partial comfort to the vertical vibrations is calculated. The paper is structured into two parts. The Part I includes all the theoretical elements required for the analysis of the dynamic response in the vehicle, while Part II introduces the results of the numerical analysis.
\end{abstract}

\section{Introduction}

During running, the railway vehicle is subjected to a permanent behaviour of vibrations, mainly generated by the track geometrical irregularities [1]. The vehicle has to have the ability to limit the behaviour of vibrations to a level where the dynamic performance is not influenced [2]. The analysis of the dynamic response in the railway vehicle to the track geometrical irregularities is an important step in the investigation of the issues about the ride quality or the ride comfort [3 - 5].

While running on a track with vertical irregularities, the axles make forced movements in a vertical plan, so that the plan of the axles in a bogie has a translation (bounce) and rotation motion (pitch) (fig. 1). For a two-bogie vehicle, the combination of the bounce and pitch motions in the plans of the axles of each bogie will result into the symmetrical and antisymmetrical motion modes of the plans of the vehicle axles (fig. 2 and fig. 3). Further on, these motions are conveyed to the suspended massed, via the suspension elements, thus exciting the symmetrical and antisymmetrical vibration modes of the railway vehicle in a vertical plan [6]. (a)

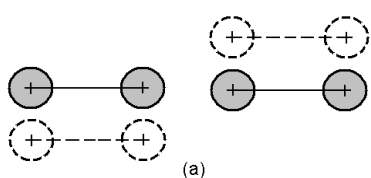

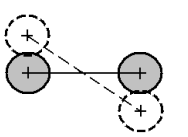

(b)

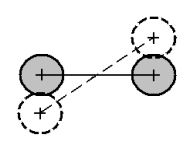

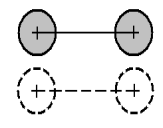

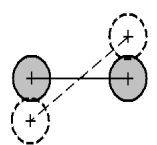

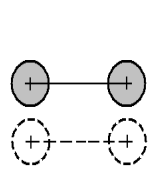
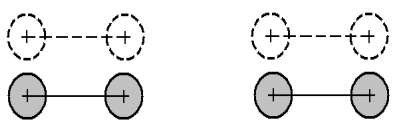

(a)
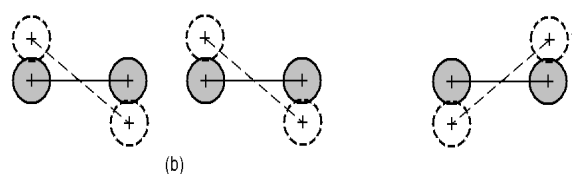

Fig. 2. The symmetrical motion modes of the planes of the vehicle's axles: (a) symmetrical bounce; (b) symmetrical pitch.
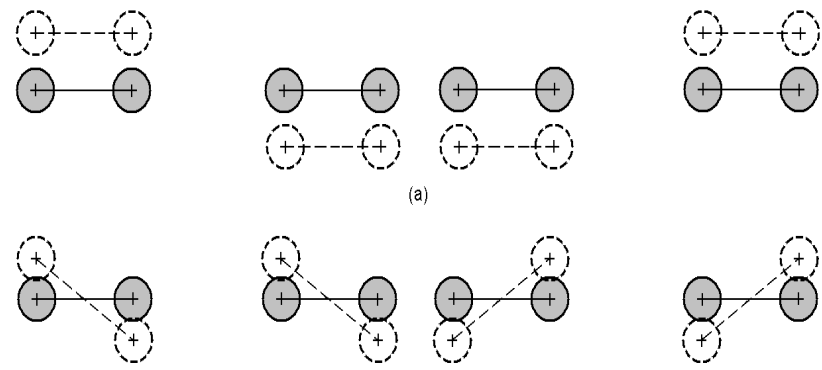

Fig. 3. The symmetrical motion modes of the planes of the vehicle's axles: (a) antisymmetrical bounce; (b) antisymmetrical pitch.

An important feature of the vertical vibrations in the railway vehicle is the geometric filtering effect of the excitation modes induced by the track vertical irregularities $[3,7-10]$. As a matter of fact, this effect is the result of the movements between the vertical motions of the axles, which depend on the distance between the axles and on the velocity. As a consequence of the geometric filtering effect in the vehicle response, a series of minimum values will All rights reserved.

\footnotetext{
* E-mail address: madalina.dumitriu@yahoo.com ISSN: 1791-2377 @ 2015 Kavala Institute of Technology.
} 
occur, as an exclusive result of how the excitations derived from the track irregularities are transmitted to the suspended masses via the axles motions, irrespective of the suspension characteristics. Under certain conditions that are solely incidental to the wavelength of the track vertical irregularity and the distance between the axles, the axles plan can only have pitch motion, since the bounce motion is not conveyed to the bogie - the plan of the axles filter the bounce. Similarly, this plan can only perform bounce motions - in this case, the pitch motion is not transmitted to the bogie the plan of the axles filters the pitch [11].

The complexity degree of the vehicle model for studying the vertical vibrations is generally established in dependence on the precision required from the results. The more complex the model is, the closer the results are to reality, but it will be more difficult to draw general conclusions regarding the basic phenomena in the vehicle dynamics. Quality and even quantity results can be acquired based on ,rigid-body' type simple mechanical models [12 -14]. Notwithstanding, the carbody modelling as a flexible body is necessary when the analysis of ride comfort is brought about [3 - 10, 16 - 18]. Even though the carbody structural vibrations are extremely complex [17, 19], the largest influence upon the dynamic behaviour of the vehicle comes from the first flexible vibration mode, whose frequency is included in the interval of interest for the vehicle vertical vibrations $0 \ldots 20 \mathrm{~Hz}[15,20]$.

The paper examines the dynamic response of the railway vehicle to the track vertical irregularities. For this purpose, the vehicle is represented by a discrete-continuous model, whose motions are described - upon the application of the modal analysis method - by two sets of four second-order ordinary differential equations, corresponding to the symmetrical and antisymmetrical vibration modes of the suspended masses. This paper focuses on the analysis of response functions in three reference points of the carbody, located at its centre and above the two bogies. They are defined by means of the carbody response functions to the symmetrical and antisymmetrical excitation modes; these functions include on their turn functions of partial response to the carbody movements for each of the symmetrical/antisymmetrical excitation modes given by the bounce/pitch in the plans of the axles. Similarly, the dynamic response of the vehicle to the track stochastic irregularities is examined and the root mean square of the acceleration and the comfort is calculated, using the relevant regulations [21 - 23].

The paper is structured into two parts. The Part I includes all the theoretical elements required for the analysis of the dynamic response in the vehicle, while Part II introduces the results of the numerical analysis.

\section{The vehicle mechanical model}

To examine the vehicle response to the track vertical irregularities, the model in fig. 4 is taken into account. This is a four-axle vehicle, two suspension levels, travelling at a constant speed $V$ along a prefectly rigid track with vertical irregularities. Such irregularities are described with the reference to each axle by functions $\mathrm{h}_{j,(j+1)}$, with $j=2 i-1$, for $i=1,2$, while mentioning that each bogie is equipped with the axles $j$ and $j+1$.

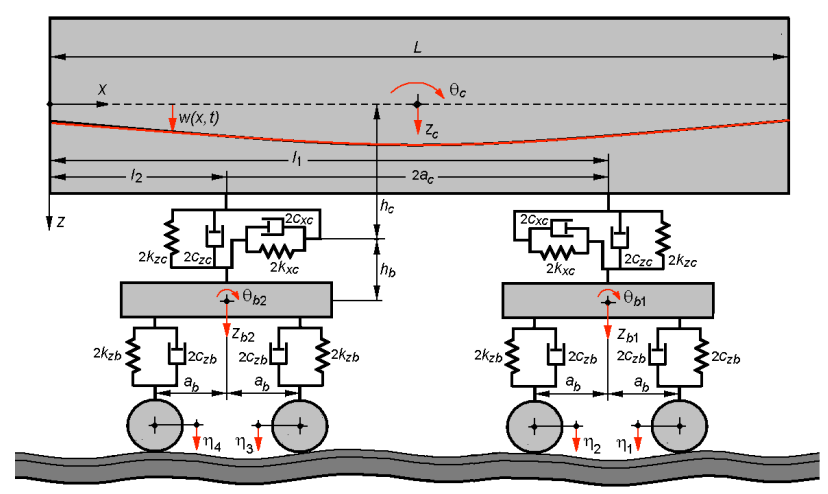

Fig. 4. The mechanical model of the vehicle/track system.

The vehicle model includes a body with distributed parameters for the carbody and many rigid bodies for the two bogies (the suspended masses) and the four axles. These bodies are connected among them via Kelvin-Voigt type systems, which help to model the suspension levels.

The carbody is represented by a free-free equivalent beam, with constant section and mass uniformly distributed, of Euler-Bernoulli type. The beam parameters are defined in terms of the carbody', such as: $L-$ beam length; $\rho_{c}=m_{c} / L-$ beam mass per length unit, where $m_{c}$ is the carbody mass; $\mu$ - structural damping coefficient; $E I$ - bending modulus, where $E$ is the longitudinal modulus of elasticity, and $I$ is the area moment of inertia of the beam transversal section. There will be taken into account the carbody rigid vibration modes - bounce $z_{c}$ and pitch $\theta_{c}$, and the first two carbody natural bending modes in a vertical plan (symmetrical and antisymmetrical). The carbody inertia reported to the rigid vibration modes is represented by mass $m_{c}$ and the mass moment of inertia $J_{c}$.

The carbody movement $w(x, t)$ comes from the superposition of the rigid vibration with the bending modes

$$
w(x, t)=z_{c}(t)+\left(x-\frac{L}{2}\right) \theta_{c}(t)+\sum_{n=2}^{3} X_{n}(x) T_{n}(t),
$$

where $T_{n}(t)$, with $n=2,3$, the coordinates of the carbody natural bending modes (symmetrical and antisymmetrical), and $X_{n}(x)$ is the eigenfunctions of the bending modes as in the relation

$$
\begin{aligned}
& X_{n}(x)=\sin \beta_{n} x+\sinh \beta_{n} x- \\
& -\frac{\sin \beta_{n} L-\sinh \beta_{n} L}{\cos \beta_{n} L-\cosh \beta_{n} L}\left(\cos \beta_{n} x+\cosh \beta_{n} x\right)
\end{aligned}
$$

with $\beta_{n}=\sqrt[4]{\omega_{n}^{2} \rho_{c} /(E I)}$ and

$\cos \beta_{n} L \cosh \beta_{n} L-1=0$,

where $\omega_{n}$ is the angular frequnecy of the bending mode $n$.

The bogies have two degrees of freedom: bounce $z_{b i}$ and pitch $\theta_{b i}$, with $i=1,2$. Each bogie has the mass $m_{b}$ and mass moment of inertia $J_{b}$. The bogie wheelbase is $2 a_{b}$, and the distance between the bogies axles is $2 a_{c}$.

The elastic and damping elements of the secondary suspension of each bogie are modelled via the Kelvin-Voigt system that operates on translation in the vertical direction, 
with the elastic constant $2 k_{z c}$, and the damping constant $2 c_{z c}$. The Kelvin-Voigt system positioned in the vertical direction at distance $h_{c}$ from the carbody neutral fiber and at distance $h_{b}$ from the bogie centre of gravity models the transmission system of the longitudinal forces between the carbody and the bogie. This has the elastic constant $2 k_{x c}$ and the damping constant $2 c_{x c}$. The primary suspension corresponding to an axle is modelled by a Kelvin-Voigt system operating on translation in the vertical direction, with the elastic constant $2 k_{z b}$, and the damping constant $2 c_{z b}$.

\section{The vehicle motion equations}

The carbody vertical motions are described by the equations of the rigid vibration modes of the carbody and of the bogies - bounce and pitch, as well as by the equations of the first two natural bending modes of the carbody - symmetrical and antisymmetrical.

The equation of motion for the carbody has the general form of

$$
\begin{aligned}
& \frac{E I \partial^{4} w(x, t)}{\partial x^{4}}+\frac{\mu I \partial^{5} w(x, t)}{\partial x^{4} \partial t}+\frac{\rho_{c} \partial^{2} w(x, t)}{\partial t^{2}}= \\
& =\sum_{i=1}^{2} F_{z c i} \delta\left(x-l_{i}\right)+\sum_{i=1}^{2} h_{c} F_{x c i} \frac{d \delta\left(x-l_{i}\right)}{d x}
\end{aligned}
$$

where $\delta($.$) is the Dirac's delta function, the distances l_{i}$ set the position of the carbody supporting points on the secondary suspension and $F_{x c i}$ and $F_{z c i}$ stand for the forces derived from the secondary suspension of the bogie $i$

$$
F_{z c i}=-2 c_{z c}\left(\frac{\partial w\left(l_{i}, t\right)}{\partial t}-\dot{z}_{b i}\right)-2 k_{z c}\left(w\left(l_{i}, t\right)-z_{b i}\right) ;
$$

$$
\begin{aligned}
& F_{x c i}=2 c_{x c}\left(h_{c} \frac{\partial^{2} w\left(l_{i}, t\right)}{\partial x \partial t}+h_{b} \dot{\theta}_{b i}\right)+ \\
& +2 k_{x c}\left(h_{c} \frac{\partial w\left(l_{i}, t\right)}{\partial x}+h_{b} \theta_{b i}\right)
\end{aligned}
$$

Upon the application of the modal analysis method and considering the orthogonality property of the eigenfunctions of the carbody bending modes, the equation of motion (4) is changed into four second-order differential equations with ordinary derivatives that describe the bounce, pitch, symmetrical and antisymmetrical bending carbody motions:

$$
\begin{aligned}
& m_{c} \ddot{z}_{c}=\sum_{i=1}^{2} F_{z c i} ; \\
& J_{c} \ddot{\theta}_{c}=\sum_{i=1}^{2} F_{z c i}\left(l_{i}-\frac{L}{2}\right)-\sum_{i=1}^{2} h_{c} F_{x c i} ; \\
& m_{m 2} \ddot{T}_{2}+c_{m 2} \dot{T}_{2}+k_{m 2} T_{2}=\sum_{i=1}^{2} F_{z c i} X_{2}\left(l_{i}\right)-\sum_{i=1}^{2} h_{c} F_{x c i} \frac{d X_{2}\left(l_{i}\right)}{d x} . \\
& m_{m 3} \ddot{T}_{3}+c_{m 3} \dot{T}_{3}+k_{m 3} T_{3}=\sum_{i=1}^{2} F_{z c i} X_{3}\left(l_{i}\right)-\sum_{i=1}^{2} h_{c} F_{x c i} \frac{d X_{3}\left(l_{i}\right)}{d x}
\end{aligned}
$$

where $m_{m 2,3}$ is the modal mass, $c_{m 2,3}$ - modal damping and $k_{m 2,3}$ - the modal stiffness corresponding to the first two natural bending modes of the carbody. They can be calculated via the below relations, for $n=2,3$ :

$$
\begin{aligned}
& k_{m n}=E I \int_{0}^{L}\left(\frac{d^{2} X_{n}}{d x^{2}}\right)^{2} d x \\
& m_{m n}=\rho_{c} \int_{0}^{L} X_{n}^{2} d x .
\end{aligned}
$$$$
c_{m n}=\mu I \int_{0}^{L}\left(\frac{d^{2} X_{n}}{d x^{2}}\right)^{2} d x
$$

The equations describing the bounce and pitch motions of the bogies are:

$m_{b} \ddot{z}_{b i}=\sum_{j=2 i-1}^{2 i} F_{z b j, j+1}-F_{z c i}$

with $i=1,2$ and $j=2 i-1$;

$J_{b} \ddot{\theta}_{b i}=a_{b} \sum_{j=2 i-1}^{2 i}(-1)^{j+1} F_{z b j}-h_{b} F_{x c i}$,

with $i=1,2$ and $j=2 i-1$,

where $F_{z b j, j+1}$ represent the forces due to the primary suspension corresponding to the axles $j$ and $(j+1)$, respectively

$$
\begin{aligned}
& F_{z b j, j+1}=-2 c_{z b}\left(\dot{z}_{b i} \pm a_{b} \dot{\theta}_{b i}-\dot{\eta}_{j, j+1}\right)- \\
& -2 k_{z b}\left(z_{b i} \pm a_{b} \theta_{b i}-\eta_{j, j+1}\right)
\end{aligned} .
$$

The analysis of the dynamic response of the vehicle can be more easily done depending on the symmetrical and antisymmetrical motions in the system. To this purpose, the coordinates of the symmetrical motions are being introduced

$$
p_{1}^{+}=z_{c} ; p_{2}^{+}=T_{2} ; p_{3}^{+}=\frac{1}{2}\left(z_{b 1}+z_{b 2}\right) ; p_{4}^{+}=\frac{1}{2}\left(\theta_{b 1}-\theta_{b 2}\right),
$$

and of the antisymmetrical ones

$$
p_{1}^{-}=\theta_{c} ; p_{2}^{-}=T_{3} ; p_{3}^{-}=\frac{1}{2}\left(z_{b 1}-z_{b 2}\right) ; p_{4}^{-}=\frac{1}{2}\left(\theta_{b 1}+\theta_{b 2}\right) \text {. }
$$

Similarly, the symmetrical excitation modes induced by the track vertical irregularities are entered

$$
\eta_{1}^{+}=\frac{\eta_{1}+\eta_{2}+\eta_{3}+\eta_{4}}{4} ; \eta_{2}^{+}=\frac{\eta_{1}-\eta_{2}-\eta_{3}+\eta_{4}}{4}
$$

while mentioning that $\eta_{1}^{+}$corresponds to the symmetrical bounce of the axles' planes, whereas $\eta_{2}^{+}$- to the symmetrical pitch of the same planes.

Likewise, for the antisymmetrical excitation modes, it is written

$\eta_{1}^{-}=\frac{\eta_{1}+\eta_{2}-\eta_{3}-\eta_{4}}{4} ; \eta_{2}^{-}=\frac{\eta_{1}-\eta_{2}+\eta_{3}-\eta_{4}}{4}$ 
where $\eta_{1}^{-}$represents the excitation mode correlated with the antisymmetrical bounce of the axles' planes and $\eta_{2}^{-}-$with the antisymmetrical pitch in the same planes.

Based on the symmetry and antisymmetry properties of the eigenfunctions in the first two vertical bending modes of the carbody, the following notations can be introduced:

$\varepsilon^{+}=X_{2}\left(l_{1}\right)=X_{2}\left(l_{2}\right) ; \varepsilon^{-}=X_{3}\left(l_{1}\right)=-X_{3}\left(l_{2}\right) ;$

$\lambda^{+}=\frac{d X_{2}\left(l_{1}\right)}{d x}=-\frac{d X_{2}\left(l_{2}\right)}{d x} ; \lambda^{-}=\frac{d X_{3}\left(l_{1}\right)}{d x}=\frac{d X_{3}\left(l_{2}\right)}{d x}$.

Upon using the above notations and adequately processing, the set comprising the equations (7) - (10) and (12) - (13), is decomposed into two independent fourequations sets, which describe the symmetrical and antisymmetrical motions of the vehicle,

$$
\mathbf{M}^{+} \ddot{\mathbf{p}}^{+}+\mathbf{C}^{+} \dot{\mathbf{p}}^{+}+\mathbf{K}^{+} \mathbf{p}^{+}=\mathbf{P} \dot{\boldsymbol{\eta}}^{+}+\mathbf{R} \boldsymbol{\eta}^{+}
$$

$\mathbf{M}^{-} \ddot{\mathbf{p}}^{-}+\mathbf{C}^{-} \dot{\mathbf{p}}^{-}+\mathbf{K}^{-} \mathbf{p}^{-}=\mathbf{P} \dot{\boldsymbol{\eta}}^{-}+\mathbf{R} \boldsymbol{\eta}^{-}$,

where $\mathbf{p}^{ \pm}=\left[\begin{array}{cccc}p_{1}^{ \pm} & p_{2}^{ \pm} & p_{3}^{ \pm} & p_{4}^{ \pm}\end{array}\right]^{T}$ stand for the vectors of the motion coordinates and $\eta^{ \pm}=\left[\begin{array}{ll}\eta_{1}^{ \pm} & \eta_{2}^{ \pm}\end{array}\right]^{T}$ - the vectors of the symmetrictial and antisymmetrical excitation modes.

The matrices $\mathbf{M}^{+}$and $\mathbf{M}^{-}$are the inertia matrices, in the form of

$\mathbf{M}^{+}=\operatorname{diag}\left(m_{c}, m_{m 2}, m_{b}, J_{b}\right) ; \mathbf{M}^{-}=\operatorname{diag}\left(J_{c}, m_{m 3}, m_{b}, J_{b}\right)$. below

The damping matrices, noted as $\mathbf{C}^{+}$and $\mathbf{C}^{-}$, write as $\mathbf{C}^{+}=\left[\begin{array}{cccc}4 c_{z c} & 4 c_{z c} \varepsilon^{+} & -4 c_{z c} & 0 \\ 4 c_{z c} \varepsilon^{+} & C_{1} & -4 c_{z c} \varepsilon^{+} & 4 c_{x c} h_{c} h_{b} \lambda^{+} \\ -2 c_{z c} & -2 c_{z c} \varepsilon^{+} & C_{2} & 0 \\ 0 & 2 c_{x c} h_{c} h_{b} \lambda^{+} & 0 & C_{3}\end{array}\right]$

$\mathbf{C}^{-}=\left[\begin{array}{cccc}C_{4} & C_{5} & -4 c_{z c} a_{c} & 4 c_{x c} h_{c} h_{b} \\ C_{5} & C_{6} & -4 c_{z c} \varepsilon^{-} & 4 c_{x c} h_{c} h_{b} \lambda^{-} \\ -2 c_{z c} a_{c} & -2 c_{z c} \varepsilon^{-} & C_{2} & 0 \\ 2 c_{x c} h_{c} h_{b} & 2 c_{x c} h_{c} h_{b} \lambda^{-} & 0 & C_{3}\end{array}\right]$,

where the following notations were used

$$
\begin{aligned}
& C_{1}=c_{m 2}+4 c_{z c}\left(\varepsilon^{+}\right)^{2}+4 c_{x c} h_{c}^{2}\left(\lambda^{+}\right)^{2} ; C_{2}=4 c_{z b}+2 c_{z c} ; \\
& C_{3}=4 c_{z b} a_{b}^{2}+2 c_{x c} h_{b}^{2} ; C_{4}=4 c_{z c} a_{c}^{2}+4 c_{x c} h_{c}^{2} \\
& C_{5}=4 c_{z c} a_{c} \varepsilon^{-}+4 c_{x c} h_{c}^{2} \lambda^{-} ; \\
& C_{6}=c_{m 3}+4 c_{z c}\left(\varepsilon^{-}\right)^{2}+4 c_{x c} h_{c}^{2}\left(\lambda^{-}\right)^{2} .
\end{aligned}
$$

The matrices $\mathrm{K}^{+}$and $\mathrm{K}^{-}$are the stiffness matrices, written as

$\mathbf{K}^{+}=\left[\begin{array}{cccc}4 k_{z c} & 4 k_{z c} \varepsilon^{+} & -4 k_{z c} & 0 \\ 4 k_{z c} \varepsilon^{+} & K_{1} & -4 k_{z c} \varepsilon^{+} & 4 k_{x c} h_{c} h_{b} \lambda^{+} \\ -2 k_{z c} & -2 k_{z c} \varepsilon^{+} & K_{2} & 0 \\ 0 & 2 k_{x c} h_{c} h_{b} \lambda^{+} & 0 & K_{3}\end{array}\right] ;$
$\mathbf{K}^{-}=\left[\begin{array}{cccc}K_{4} & K_{5} & -4 k_{z c} a_{c} & 4 k_{x c} h_{c} h_{b} \\ K_{5} & K_{6} & -4 k_{z c} \varepsilon^{-} & 4 k_{x c} h_{c} h_{b} \lambda^{-} \\ -2 k_{z c} a_{c} & -2 k_{z c} \varepsilon^{-} & K_{2} & 0 \\ 2 k_{x c} h_{c} h_{b} & 2 k_{x c} h_{c} h_{b} \lambda^{-} & 0 & K_{3}\end{array}\right]$,

with the subsequent notations:

$$
\begin{aligned}
& K_{1}=k_{m 2}+4 k_{z c}\left(\varepsilon^{+}\right)^{2}+4 k_{x c} h_{c}^{2}\left(\lambda^{+}\right)^{2} ; K_{2}=4 k_{z b}+2 k_{z c} ; \\
& K_{3}=4 k_{z b} a_{b}^{2}+2 k_{x c} h_{b}^{2} ; K_{4}=4 k_{z c} a_{c}^{2}+4 k_{x c} h_{c}^{2} ; \\
& K_{5}=4 k_{z c} a_{c} \varepsilon^{-}+4 k_{x c} h_{c}^{2} \lambda^{-} ; \\
& K_{6}=k_{m 3}+4 k_{z c}\left(\varepsilon^{-}\right)^{2}+4 k_{x c} h_{c}^{2}\left(\lambda^{-}\right)^{2}
\end{aligned}
$$

In terms of the vectors $\mathbf{P}$ and $\mathbf{R}$, they write as

$$
\mathbf{P}=\left[\begin{array}{cc}
0 & 0 \\
0 & 0 \\
4 c_{z b} & 0 \\
0 & 4 c_{z b} a_{b}
\end{array}\right] ; \mathbf{R}=\left[\begin{array}{cc}
0 & 0 \\
0 & 0 \\
4 k_{z b} & 0 \\
0 & 4 k_{z b} a_{b}
\end{array}\right]
$$

\section{The vehicle response functions}

Further on, it is considered that the track vertical irregularities are in a harmonic shape with the wavelength $\Lambda$ and amplitude $\eta_{0}$. With the reference to each axle, the vertical irregularities of the track can be written as

$$
\begin{aligned}
& \eta_{1,2}(x)=\eta_{0} \cos \frac{2 \pi}{\Lambda}\left(x+a_{c} \pm a_{b}\right) \\
& \eta_{3,4}(x)=\eta_{0} \cos \frac{2 \pi}{\Lambda}\left(x-a_{c} \mp a_{b}\right),
\end{aligned}
$$

where $x=V t$ is the coordinate of the carbody centre.

The functions $\eta_{j, j+1}$, with $j=2 i-1$ for $i=1,2$, can be expressed as time harmonic functions

$$
\begin{aligned}
& \eta_{1,2}(x)=\eta_{0} \cos \omega\left(t+\frac{a_{c} \pm a_{b}}{V}\right) ; \\
& \eta_{3,4}(x)=\eta_{0} \cos \omega\left(t-\frac{a_{c} \mp a_{b}}{V}\right),
\end{aligned}
$$


in which $\mathrm{w}=2 \pi V / \Lambda$ means the angular frequency induced by the track excitation.

As for the vehicle response, this is assumed to be harmonic, with the same frequency as the track excitation induced frequency. The coordinates describing the symmetrical and antisymmetrical motions of the vehicle are written under the general form as

$p_{k}^{ \pm}(t)=p_{k}^{ \pm} \cos \left(\omega t+\phi_{k}^{ \pm}\right)$

with $k=1 \div 4$,

where $p_{k}^{ \pm}$is the amplitude, and $\phi_{k}^{ \pm}$represents the phase of the coordinate $k$ compared to the track vertical irregularities with respect to the vehicle centre.

In addition, the complex values associated with the real ones, for $i^{2}=-1$ :

- for the track vertical irregularities with the reference to the axles

$\bar{\eta}_{j, j+1}(t)=\bar{\eta}_{j, j+1} e^{i \omega t}$

for $j=2 i-1$ and $i=1,2$;

- for the coordinates of the vehicle motions

$\bar{p}_{k}^{ \pm}(t)=\bar{p}_{k}^{ \pm} e^{i \omega t}$,

where the complex amplitudes of the track irregularities with the reference to the axles are in the form of

$\bar{\eta}_{1,2}=\eta_{0} e^{i \omega\left(t+\frac{a_{c} \pm a_{b}}{V}\right)} ; \bar{\eta}_{3,4}=\eta_{0} e^{i \omega\left(t-\frac{a_{c} \mp a_{b}}{V}\right)}$

while the complex amplitude of the coordinate $k$ writes as

$\vec{p}_{k}^{ \pm}=p_{k}^{ \pm} e^{i \phi_{k}}$

Similarly, starting from relations (17) and (18), the below equations are

$$
\begin{aligned}
& \bar{\eta}_{1,2}^{+}(t)=\frac{\bar{\eta}_{1} \pm \bar{\eta}_{2} \pm \bar{\eta}_{3}+\bar{\eta}_{4}}{4} e^{i \omega t}=\frac{1}{4} \eta_{0} \bar{H}_{f 1,2}^{+} e^{i \omega t} ; \\
& \bar{\eta}_{1,2}^{-}(t)=\frac{\bar{\eta}_{1} \pm \bar{\eta}_{2} \mp \bar{\eta}_{3}-\bar{\eta}_{4}}{4} e^{i \omega t}=\frac{1}{4} \eta_{0} \bar{H}_{f 1,2}^{-} e^{i \omega t} ;
\end{aligned}
$$

where

$\bar{H}_{f 1,2}^{+}=e^{i \omega \frac{a_{c}+a_{b}}{V}} \pm e^{i \omega \frac{a_{c}-a_{b}}{V}} \pm e^{-i \omega \frac{a_{c}-a_{b}}{V}}+e^{-i \omega \frac{a_{c}+a_{b}}{V}} ;$

$\bar{H}_{f 1,2}^{-}=e^{i \omega \frac{a_{c}+a_{b}}{V}} \pm e^{i \omega \frac{a_{c}-a_{b}}{V}} \mp e^{-i \omega \frac{a_{c}-a_{b}}{V}}-e^{-i \omega \frac{a_{c}+a_{b}}{V}}$.

are the characteristics of geometric filtering effect of the symmetrical and antisymmetrical excitation modes.

The vectors of the symmetrical and antisymmetrical excitation modes write as $\bar{\eta}^{+}(t)=\eta_{0} \overline{\mathbf{H}}_{f}^{+} e^{i \omega t}=\eta_{0}\left[\quad \bar{H}_{f 1}^{+} \quad \bar{H}_{f 2}^{+}\right]^{T} e^{i \omega t}$

$\bar{\eta}^{-}(t)=\eta_{0} \overline{\mathbf{H}}_{f}^{-} e^{i \omega t}=\eta_{0}\left[\bar{H}_{f 1}^{-} \quad \bar{H}_{f 2}^{-}\right]^{T} e^{i \omega t}$,

where $\overline{\mathbf{H}}_{f}^{ \pm}$is the column vectors of the geometric filtering effect characteristics in the symmetrical and antisymmetrical excitation modes.

The vectors of the coordinates of the car body and bogies movement thus become:

$\overrightarrow{\mathbf{p}}^{ \pm}(t)=\overrightarrow{\mathbf{p}}^{ \pm} \exp (i \omega t)$

where $\overline{\mathbf{p}}^{ \pm}=\left[\begin{array}{cccc}\bar{p}_{1}^{ \pm} & \bar{p}_{2}^{ \pm} & \bar{p}_{3}^{ \pm} & \bar{p}_{4}^{ \pm}\end{array}\right]^{T}$ is the vector of the complex amplitudes of the movements.

The equations of motion (21) and (22) of the vehicle are as:

$\left(-\omega^{2} \mathbf{M}^{+}+\mathbf{K}^{+}+i \omega \mathbf{C}^{+}\right) \overline{\mathbf{p}}^{+}=(i \omega \mathbf{P}+\mathbf{R}) \eta_{0} \overline{\mathbf{H}}_{f}^{+}$

$\left(-\omega^{2} \mathbf{M}^{-}+\mathbf{K}^{-}+i \omega \mathbf{C}^{-}\right) \overline{\mathbf{p}}^{-}=(i \omega \mathbf{P}+\mathbf{R}) \eta_{0} \overline{\mathbf{H}}_{f}^{-}$.

Further on, the response functions of the vehicle to the track induced excitations can be established. The vector of the response functions to the symmetrical and antisymmetrical excitations will be calculated as below

$\overline{\mathbf{H}}^{+}=\overline{\mathbf{p}}^{+} / \eta_{0} ; \overline{\mathbf{H}}^{-}=\overline{\mathbf{p}}^{-} / \eta_{0}$.

The relations above can be rewritten as

$\overline{\mathbf{H}}^{+}=\overline{\mathbf{H}}_{p}^{+} \overline{\mathbf{H}}_{f}^{+} ; \overline{\mathbf{H}}^{-}=\overline{\mathbf{H}}_{p}^{-} \overline{\mathbf{H}}_{f}^{-}$,

where $\quad \overline{\mathbf{H}}_{p}^{+}=\left[-\omega^{2} \mathbf{M}^{+}+\mathbf{K}^{+}+i \omega \mathbf{C}^{+}\right]^{-1}[i \omega \mathbf{P}+\mathbf{R}]$ can be named the matrix of the vehicle functions of partial response to the two symmetrical excitation modes, whereas $\overline{\mathbf{H}}_{p}^{-}=\left[-\omega^{2} \mathbf{M}^{-}+\mathbf{K}^{-}+i \omega \mathbf{C}^{-}\right]^{-1}[i \omega \mathbf{P}+\mathbf{R}]-$ is the matrix of the vehicle functions of partial response to the two antisymmetrical excitation modes. These two matrices are as below

$\overline{\mathbf{H}}_{p}^{+}=\left[\begin{array}{cc}\bar{H}_{p 11}^{+} & \bar{H}_{p 12}^{+} \\ \bar{H}_{p 21}^{+} & \bar{H}_{p 22}^{+} \\ \bar{H}_{p 31}^{+} & \bar{H}_{p 32}^{+} \\ \bar{H}_{p 41}^{+} & \bar{H}_{p 42}^{+}\end{array}\right] ; \mathbf{H}_{p}^{-}=\left[\begin{array}{cc}\bar{H}_{p 11}^{-} & \bar{H}_{p 12}^{-} \\ \bar{H}_{p 21}^{-} & \bar{H}_{p 22}^{-} \\ \bar{H}_{p 31}^{-} & \bar{H}_{p 32}^{-} \\ \bar{H}_{p 41}^{-} & \bar{H}_{p 42}^{-}\end{array}\right]$.

Based on the latest relations, the response functions of the vehicle carbody can be determined for the symmetrical and antisymmetrical excitation modes. Thus, the response function corresponding to the carbody bounce motion is given by the relation

$\bar{H}_{1}^{+}=\bar{H}_{p 11}^{+} \bar{H}_{f 1}^{+}+\bar{H}_{p 12}^{+} \bar{H}_{f 2}^{+}$, 
where the functions of partial response for the carbody bounce motion excited by the symmetrical bounce of the axles' planes, $\bar{H}_{p 11}^{+}$, and by the symmetrical pitch of the axles' planes, $\bar{H}_{p 12}^{+}$, can be found.

The response function for the carbody symmetrical bending can be similarly calculated:

$$
\bar{H}_{2}^{+}=\bar{H}_{p 21}^{+} \bar{H}_{f 1}^{+}+\bar{H}_{p 22}^{+} \bar{H}_{f 2}^{+},
$$

where $\bar{H}_{p 21}^{+}$is the function of partial response corresponding to the carbody symmetrical bending excited by the symmetrical bounce of the axles' planes, and $\bar{H}_{p 22}^{+}$the function of partial response corresponding to the carbody symmetrical bending excited by the symmetrical pitch of the axles' planes.

In terms of the response functions of the vehicle carbody to the antisymmetrical excitation modes, they write as:

- for the carbody pitch motion

$\bar{H}_{1}^{-}=\bar{H}_{p 11}^{-} \bar{H}_{f 1}^{-}+\bar{H}_{p 12}^{-} \bar{H}_{f 2}^{-}$

- for the car body antisymmetrical bending

$\bar{H}_{2}^{-}=\bar{H}_{p 21}^{-} \bar{H}_{f 1}^{-}+\bar{H}_{p 22}^{-} \bar{H}_{f 2}^{-}$,

where $\bar{H}_{p 11}^{-}$and $\bar{H}_{p 12}^{-}$stand for the functions of partial response that correspond to the carbody pitch motion excited by the antisymmetrical bounce and pitch of the axles' planes; $\bar{H}_{p 21}^{-}$and $\bar{H}_{p 22}^{-}$are the functions of partial response correlated with carbody antisymmetrical bending excited by the antisymmetrical bounce and pitch of the axles' planes.

To calculate the response functions in any point of abscissa $x$, located on the carbody longitudinal axis, the relation below holds

$\bar{H}_{c}=\bar{H}_{1}^{+}+\left(\frac{L}{2}-x\right) \bar{H}_{1}^{-}+X_{2}(x) \bar{H}_{2}^{+}+X_{3}(x) \bar{H}_{2}^{-}$,

that can be applied for three carbody reference points, thence:

- at the carbody centre,

$$
\bar{H}_{c m}=\bar{H}_{1}^{+}+X_{2}(L / 2) \bar{H}_{2}^{+}
$$

or

$$
\bar{H}_{c m}=\bar{H}_{c m \eta_{1}^{+}} \bar{H}_{f 1}^{+}+\bar{H}_{c m \eta_{2}^{+}} \bar{H}_{f 2}^{+},
$$

where

$$
\bar{H}_{c m \eta_{1}^{+}}=\bar{H}_{p 11}^{+}+X_{2}(L / 2) \bar{H}_{p 21}^{+}
$$

is the function of partial response at the carbody centre to the excitation due to the symmetrical bounce of the axles' planes, and

$$
\bar{H}_{c m \eta_{2}^{+}}=\bar{H}_{p 12}^{+}+X_{2}(L / 2) \bar{H}_{p 22}^{+}
$$

is the function of partial response at the carbody centre to the excitation due to the symmetrical pitch of the axles' planes;

- above the bogies,

$\bar{H}_{c b_{1,2}}=\bar{H}_{1}^{+} \pm a_{c} \bar{H}_{1}^{-}+X_{2}\left(l_{1,2}\right) \bar{H}_{2}^{+}+X_{3}\left(l_{1,2}\right) \bar{H}_{2}^{-}$,

or

$$
\begin{aligned}
& \bar{H}_{c b_{1,2}}=\bar{H}_{c b_{1,2} \eta_{1}^{+}} \bar{H}_{f 1}^{+}+\bar{H}_{c b_{1,2} \eta_{2}^{+}} \bar{H}_{f 2}^{+}+ \\
& +\bar{H}_{c b_{1,2} \eta_{2}^{+}} \bar{H}_{f 1}^{-}+\bar{H}_{c b_{1,2} \eta_{2}^{-}} \bar{H}_{f 2}^{-}
\end{aligned},
$$

where the notations below

$$
\bar{H}_{c b_{1,2} \eta_{1}^{+}}=\bar{H}_{p 11}^{+}+X_{2}\left(l_{1,2}\right) \bar{H}_{p 21}^{+}
$$

$\bar{H}_{c b_{1,2} \eta_{2}^{+}}=\bar{H}_{p 12}^{+}+X_{2}\left(l_{1,2}\right) \bar{H}_{p 22}^{+}$

are the functions of partial response of the carbody above the bogies to the excitation coming from the symmetrical bounce and symmetrical pitch, respectively, of the axles' planes, and

$$
\bar{H}_{c b_{1,2} \eta_{1}^{-}}= \pm a_{c} \bar{H}_{p 11}^{-}+X_{3}\left(l_{1,2}\right) \bar{H}_{p 21}^{-}
$$

$\bar{H}_{c b_{1,2} \eta_{2}^{-}}= \pm a_{c} \bar{H}_{p 12}^{-}+X_{3}\left(l_{1,2}\right) \bar{H}_{p 22}^{-}$,

are the functions of partial response of the carbody above the bogies to the excitations due to the antisymmetrical bounce and pitch of the axles' planes.

In dependence on the symmetry and antisymmetry properties of the natural functions of the symmetrical and antisymmetrical carbody bending and according to relations (19), the partial response functions of the carbody in the points above the bogies write as

$$
\begin{aligned}
& \bar{H}_{c b \eta_{1}^{+}}=\bar{H}_{c b_{1} \eta_{1}^{+}}=\bar{H}_{c b_{2} \eta_{1}^{+}}=\bar{H}_{p 11}^{+}+\varepsilon^{+} \bar{H}_{p 21}^{+} ; \\
& \bar{H}_{c b \eta_{2}^{+}}=\bar{H}_{c b_{1} \eta_{2}^{+}}=\bar{H}_{c b_{2} \eta_{2}^{+}}=\bar{H}_{p 12}^{+}+\varepsilon^{+} \bar{H}_{p 22}^{+} ; \\
& \bar{H}_{c b \eta_{1}^{-}}=\bar{H}_{c b_{1} \eta_{1}^{-}}=-\bar{H}_{c b_{2} \eta_{1}^{-}}=a_{c} \bar{H}_{p 11}^{-}+\varepsilon^{-} \bar{H}_{p 21}^{-} ;
\end{aligned}
$$

$\bar{H}_{c b \eta_{2}^{-}}=\bar{H}_{c b_{1} \eta_{2}^{-}}=-\bar{H}_{c b_{2} \eta_{2}^{-}}=a_{c} \bar{H}_{p 12}^{-}+\varepsilon^{-} \bar{H}_{p 22}^{-}$.

According to the latest notations, relation (52) becomes

$\bar{H}_{c b 1,2}=\bar{H}_{c b \eta_{1}^{+}} \bar{H}_{f 1}^{+}+\bar{H}_{c b \eta_{2}^{+}} \bar{H}_{f 2}^{+} \pm \bar{H}_{c b \eta_{1}^{-}} \bar{H}_{f 1}^{-} \pm \bar{H}_{c b \eta_{2}^{-}} \bar{H}_{f 2}^{-}$ 


\section{The vehicle dynamic response to the track stochastic irregularities}

Further on, the track vertical irregularities are considered to represent a stationary stochastic process, which can be described via the power spectral density. The theoretical curve of the power spectral density is representative for the average statistical properties of the European railway, as in the relation [21]

$S(\Omega)=\frac{A \Omega_{c}^{2}}{\left(\Omega^{2}+\Omega_{r}^{2}\right)\left(\Omega^{2}+\Omega_{c}^{2}\right)}$,

where $\Omega$ is the wavelength, $\Omega_{c}=0.8246 \mathrm{rad} / \mathrm{m}, \Omega_{r}=0.0206$ $\mathrm{rad} / \mathrm{m}$, and $A$ is a coefficient depending on the track quality. For a high level quality track, $A=4.032 \cdot 10^{-7} \mathrm{radm}$, whereas for a low level quality, the coefficient $A$ is $1.080 \cdot 10^{-6} \mathrm{radm}$.

As a function of the angular frequency $\omega=V \Omega$, the power spectral density of the track irregularities can be written as in the general relation

$$
G(\omega)=S(\omega / V)
$$

What results is the power spectral density of the track irregularities in the form of

$$
G(\omega)=\frac{A \Omega_{c}^{2} V^{3}}{\left[\omega^{2}+\left(V \Omega_{c}\right)^{2}\right]\left[\omega^{2}+\left(V \Omega_{r}\right)^{2}\right]} .
$$

Starting from the response functions of the vehicle carbody and the spectrum of the track irregularities, the power spectral density of the carbody vertical movement is calculated, as in the general relation

$$
G_{c}=G(\omega)\left|\bar{H}_{c}\right|^{2}
$$

Upon applying the above equation, the result will be the power spectral density in the reference point at the carbody centre and in the points above the bogies, respectively:

$$
G_{c m}=G(\omega)\left|\bar{H}_{c m}\right|^{2} ; G_{c b_{1,2}}=G(\omega)\left|\bar{H}_{c b_{1,2}}\right|^{2} \text {. }
$$

The power spectral density of the carbody acceleration is established from the below equation:

$$
G_{c a}=\omega^{4} G(\omega)\left|\bar{H}_{c}\right|^{2},
$$

which can be also written for the three reference carbody points by a suitable replacement of the response functions with the particular relations in the previous section.

Based on vehicle dynamic response, expressed in the form of the power spectral density of the carbody acceleration, the root mean square acceleration and the comfort index can be further calculated, values that count in evaluating the ride quality and the ride comfort.

\section{Evaluating the ride quality and ride comfort}

The ride quality in a railway vehicle is evaluated by means of the acceleration root mean square [22]. Starting from the power spectral density of the acceleration, the root mean square of the carbody vertical acceleration can be established via the formula

$$
a_{c}=\sqrt{\frac{1}{\pi} \int_{0}^{\infty} G_{c a} d \omega}
$$

To evaluate the ride comfort in the vertical direction, the partial comfort index is used, which is calculated with the relation [23]

$$
N_{M V}=6 a_{c 95}^{W_{a b}}
$$

where $a_{c}$ is the root mean square of the vertical acceleration, 95 refers to the quantile of order $95 \%$, and $W_{a b}=W_{a} \cdot W_{b}$ represents the weight filter of the accelerations in the vertical direction.
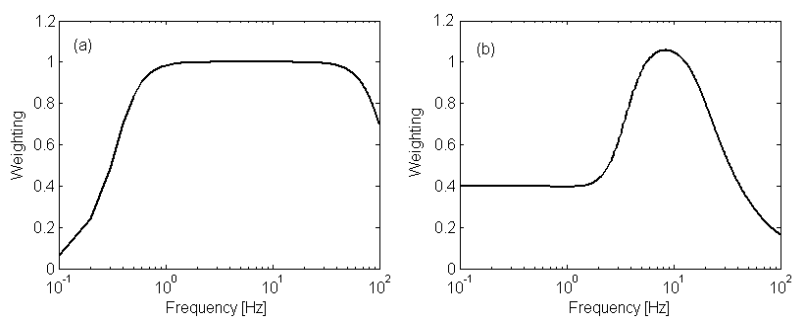

Fig. 5. The transfer functions of the weighting filters: (a) for filter $W_{a}$; (b) for filter $W_{b}$.

The filter $W_{a}$ is a band-pass type filter, with the following transfer function (fig. 5, (a))

$$
H_{a}(s)=\frac{s^{2}\left(2 \pi f_{2}\right)^{2}}{\left[s^{2}+\frac{2 \pi f_{1}}{Q_{1}} s+\left(2 \pi f_{1}\right)^{2}\right]\left[s^{2}+\frac{2 \pi f_{2}}{Q_{1}} s+\left(2 \pi f_{2}\right)^{2}\right]}
$$

with $f_{1}=0.4 \mathrm{~Hz}, f_{2}=100 \mathrm{~Hz}$ and $Q_{1}=0.71$.

The weighting filter $W_{b}$, which takes into account the higher human sensitivity to the vertical vibrations within the frequencies ranging from 3 to $13 \mathrm{~Hz}$, has the transfer function in the form of (fig. 5, (b))

$$
H_{b}(s)=\frac{\left(s+2 \pi f_{3}\right) \cdot\left[s^{2}+\frac{2 \pi f_{5}}{Q_{3}} s+\left(2 \pi f_{5}\right)^{2}\right] 2 \pi K f_{4}^{2} f_{6}^{2}}{\left[s^{2}+\frac{2 \pi f_{4}}{Q_{2}} s+\left(2 \pi f_{4}\right)^{2}\right]\left[s^{2}+\frac{2 \pi f_{6}}{Q_{4}} s+\left(2 \pi f_{6}\right)^{2}\right] f_{3} f_{5}^{2}}
$$

where $f_{3}=16 \mathrm{~Hz}, f_{4}=16 \mathrm{~Hz}, f_{5}=2.5 \mathrm{~Hz}, f_{6}=4 \mathrm{~Hz}, Q_{2}=$ $0.63, Q_{4}=0.8, K=0.4$ and $s=\mathrm{i} \omega$ (with $\mathrm{i}^{2}=-1$ ).

When adopting the hypothesis that the vertical accelerations have a Gaussian distribution with the null mean value and considering the relation (68) to calculate the root mean square acceleration, the following relation for the comfort index is derived 


$$
N_{M V}=6 \Phi^{-1}(0,95) \sqrt{\frac{1}{\pi} \int_{0}^{\infty} G_{c a}\left|\bar{H}_{a b}\right|^{2} d \omega},
$$

where $\Phi^{-1}(0,95)$ represents the quantile of the standard Gaussian distribution with the probability of $95 \%$.

\section{Summary}

The Part I of this paper includes the mechanical model of the vehicle and the equations of motion, the response functions, as well as the theoretical elements required for the analysis of the dynamic response of the railway vehicle to the track vertical stochastic irregularities.

To study the vertical vibrations of the vehicle, this is represented via a discrete-continuous model with eight degrees of freedom. The excitation modes induced by the track vertical irregularities come, on the one hand, from the symmetrical bounce and pitch of the axles' planes - the symmetrical excitation modes - and, on the other hand, by the antisymmetrical bounce and pitch of the axles' planes the antisymmetrical excitation modes. The equations of motion are as such processed that the symmetrical and antisymmetrical motions of the vehicle be described via two independent sets of equations. Similarly, the response functions of the vehicle carbody are separately established for the symmetrical and for the antisymmetrical excitation modes. They include the partial response functions for the carbody movements for each of the four excitation modes. These functions serve as a basis of calculation for the response functions in three reference points of the carbody at the centre and above the two bogies.

The dynamic response of the vehicle to the track stochastic irregularities is in the form of the power spectral density of the carbody acceleration, based on which the evaluation quantities of the ride quality and ride comfort the root square mean of acceleration and comfort index - are calculated.

\section{References}

1. I. Sebeşan, T. Mazilu, Vibraţiile vehiculelor feroviare (Vibrations of the railway vehicles), MatrixRom, București, 2010.

2. V.K. Garg, R.V. Dukkipati, Dynamics of railway vehicle systems, Academic Press, New York. 1984.

3. J. Zhou, R. Goodall, L. Ren, H. Zhang, Influences of car body vertical flexibility on ride quality of passenger railway vehicles, Proceedings of the Institution of Mechanical Engineers, Part F: Journal of Rail and Rapid Transit, 223, 461-471, 2009.

4 J. Zhou, G. Shen, H. Zhang, L. Ren, Application of modal parameters on ride quality improvement of railway vehicles, Vehicle System Dynamics, 46, Supplement, 629-641, 2008.

5. M. Dumitriu, Evaluation of the comfort index in railway vehicles depending on the vertical suspension, Annals of Faculty Engineering Hunedoara - International Journal of Engineering, XI, 4, 23-32, 2013.

6. M. Dumitriu, Considerations on the excitation mechanism of the vertical vibration symmetrical and antisymmetrical modes in railway vehicles, Annals of the University of Petroşani, Mechanical Engineering, 15, 58-71, 2013.

7. D. Gong, W. Sun, J. Zhou, X. Xie, Analysis on the vertical coupled vibration between bogies and metro car body, Procedia Engineering, 16, 825-831, 2011.

8. D. Gong, Y.J. Gu, Y.J. Song, J. Zhou, Study on geometry filtering phenomenon and flexible car body resonant vibration of articulated trains, Advanced Materials Research, 787, 542-547, 2013.

9. J. Zhou, S. Wenjing, Analysis on geometric filtering phenomenon and flexible car body resonant, Vibration of Railway Vehicles, Journal of Tongji University, Natural Science, 37, 12,1653$1657,2009$.

10. M. Dumitriu, Geometric filtering effect of vertical vibrations of railway vehicles, Analele Universităţii "Eftimie Murgu" Resiţa, 1, 48-61, 2012.

11. M. Dumitriu, Considerations on the geometric filtering effect of the bounce and pitch movements in railway vehicles, Annals of Faculty Engineering Hunedoara - International Journal of Engineering, XII, 3, 155-164, 2014.

12. G.R.M Mastinu, M. Gobbi, G.D. Pace, Analytical formulae for the design of a railway vehicle suspension system, Proceedings of the Institution of Mechanical Engineers, Part F: Journal of Rail and Rapid Transit, 215, 683-698, 2001.

13. M. Dumitriu, On the assessment of the vertical vibration behaviour of a railway vehicle, Romanian Journal of Acoustics and Vibration, VIII, 2, 131-138, 2011.

14. R.C. Sharma, Parametric analysis of rail vehicle parameters influencing ride behavior, International Journal of Engineering, Science and Technology, 3, 8, 54-65, 2011.

15 F. Cheli, R. Corradi, On rail vehicle vibrations induced by track unevenness: Analysis of the excitation mechanism, Journal of Sound and Vibration, 330, 3744-3765, 2011.
16. G. Schandl, P. Lugner, C. Benatzky, M. Kozek, M. Stribersk, Comfort enhancement by an active vibration reduction system for a flexible railway car body, Vehicle System Dynamics, 45, 9, 835847, 2007.

17. T. Tomioka, T. Takigami, Y. Suzuki, Numerical analysis of threedimensional flexural vibration of railway vehicle car body, Vehicle System Dynamics, 44, Supplement, 272-285, 2006.

18. M. Dumitriu, Modelling of vehicle-track system to study the vertical vibrations, Annals of the "Constantin Brâncuşi”" University of Târgiu Jiu, 4, 53-64, 2012.

19. P. Carlbom, Carbody and Passengers in Rail Vehicle Dynamics, Doctoral Thesis, Division of Railway Technology, Department of Vehicle Engineering, Royal Institute of Technology (KTH), Stockholm, Sweden, 2000.

20. G. Diana, F. Cheli, A. Collina, R. Corradi, S. Melzi, The development of a numerical model for railway vehicles comfort assessment through comparison with experimental measurements, Vehicle System Dynamics, 38, 3, 165- 183, 2002.

21. ORE B 176/1989. Bogies with steered or steering wheelsets, Report No. 1: Specifications and preliminary studies, Vol. 2. Specification for a bogie with improved curving characteristics.

22. UIC 518 Leaflet - 4th edition - Sept. 2009. Testing and approval of railway vehicles from the point of view of their dynamic behaviour - Safety - Track fatigue - Running behaviour.

23. ENV 12299/1997, Railway applications ride comfort for passengers measurement and evaluation. 